Article

\title{
Proposal for the Formalization of Dialectical Logic
}

\author{
José Luis Usó-Doménech ${ }^{1}$, Josué Antonio Nescolarde-Selva ${ }^{1, *}$ and Lorena Segura-Abad ${ }^{2}$ \\ 1 Department of Applied Mathematics, University of Alicante, Alicante 03690, Spain; kishukaze@hotmail.com \\ 2 Department of Mathematics, University of Alicante, Alicante 03690, Spain; lorena.segura.abad@gmail.com \\ * Correspondence: josue.selva@ua.es; Tel.: +34-680-418-381; Fax: +34-965-909-707
}

Academic Editor: Indranil SenGupta

Received: 18 August 2016; Accepted: 6 December 2016; Published: 11 December 2016

\begin{abstract}
Classical logic is typically concerned with abstract analysis. The problem for a synthetic logic is to transcend and unify available data to reconstruct the object as a totality. Three rules are proposed to pass from classic logic to synthetic logic. We present the category logic of qualitative opposition using examples from various sciences. This logic has been defined to include the neuter as part of qualitative opposition. The application of these rules to qualitative opposition, and, in particular, its neuter, demonstrated that a synthetic logic allows the truth of some contradictions. This synthetic logic is dialectical with a multi-valued logic, which gives every proposition a truth value in the interval $[0,1]$ that is the square of the modulus of a complex number. In this dialectical logic, contradictions of the neuter of an opposition may be true.
\end{abstract}

Keywords: analysis; contradiction; dialectics; neuter; opposition; quality; quantity; synthesis

\section{Aristotelian or Analytical Logic vs. Dialectic or Synthetic Logic}

From the cognitive flow where consciousness grasps neither itself nor the outside world, in which needs, attitudes, trends, movements, and internal and external perceptions form an undifferentiated, unreflective dynamic, rational thinking emerges with analyses based on the perception of identities and differences fixed and structured by language. The results of the analyses are abstract concepts whose content is identical to a set of different objects. Abstract concepts are arranged hierarchically, general and particular, but their main characteristic is to be general, we might "especially" wish to ask them to be "less general". At this early (required) stage of scientific thinking, reasoning conforms to an analytical logic whose prototype is Aristotelian logic. The basic principles of Aristotelian classic (analytical) logic include the following:

(1) Principle of Non-Contradiction (PNC): It is impossible that something is true $(P)$ and false $(\neg P)$ at the same time, though both can be false $\neg(P \wedge \neg P)$.

(2) Principle of Excluded Middle (PEM): Either a proposition is true or its negation is true: alternatively put, $P \vee \neg P$. It is also called tertium non datur, meaning that the third possibility is not given.

(3) Principle of Exclusive Disjunction for Contradictories (PEDC): exactly one is true; exactly one is false.

Let $\boldsymbol{P}$ be a proposition and $\neg P$ its negation. From Morgan's Theorem, PNC can be transformed into PEM and vice versa, but this only demonstrates that Morgan's Theorem estimates PEDC. Logics that deny PEM must deny some forms of Morgan's Theorem. PNC and PEM do not need to be equivalent in $n$-valued logics when $n>2$, although the principles must be reformulated for these logics and could seem very different. Even in two-valued logics, these three formulas are different as soon as we replace $P$ and $\neg P$ with $P$ and $Q$. The relationships that they affirm are only equivalent in a special case: when the relations of contradiction are affirmed. If we used a two-value logical standard, the three principles do not appear in the form of axioms. The three principles can be demonstrated to be logical, but any such proof would be viciously circular. 
The PNC is a fundamental principle of classical or analytical logic. In this logic, any contradiction should be rejected as false, which is expressed by the expression: $\forall P, v(P \wedge \neg P)=0$. The algebraic reduction for this expression is: $p(1-p)=0$, an equation which admits as solutions $p=0 ; p=1$, i.e., a single solution that retains Aristotelian logic.

Many classical logic principles (PNC, PEM, PEDC, etc.) are invalidated in modern logics. For example, PEM is invalidated in neutrosophic logic (neutrosophy is a generalization of dialectics, i.e., while in dialectics, there is a dynamic of opposites, in neutrosophy, there is a dynamic of opposites and the neutral between them because that what is happening in our real world. For example, if two countries, A and B, go to war against each other-thus A and B are the opposites - then some neutral countries interfere in the war, helping one side or another. Hence, neutrosophy is better applied to our world than dialectics [1], not only by the Principle of the Included Middle, but also by the Principle of Multiple-Included Middle [2].

The synthesis comes after the analysis: the thought that wants to materialize already has all the analytical data, and it does not pretend to ignore nor reject the truths of abstract logic, but persists, however, to assume them. Returning to both the object itself and analytical truths, however, thought brings together and unifies the operations of abstraction that have elaborated these truths, at the cost of countless rejections of peculiarities, so that it will also bring together and unify the thinking that will illuminate its object as a whole organized totality. The totality constructed in thought is, in general, not static but a dynamic becoming, an emergence of knowledge. The principle of totality is the fundamental principle of dialectical logic, and we use synthetic logic and dialectic logic interchangeably here, ignoring their differences.

We enumerate three elementary rules of this kind of logic:

(1) Keep analytic truths.

(2) Rule of unity or aggregation: with synthesis setting itself the task to recreate the unity of the object, it will return to the totality of the attributes that the analysis contained and dispersed in parts; all that is predictable as parts of the object will become predicable to a whole. Therefore, in synthetic logic, being is the only predicate.

(3) Rule of the resulting quality or the totality.

Following Priest [3-7], we will say that a logical system is paraconsistent, if and only if its relation of logical consequence is not "explosive", i.e., iff it is not the case that for every formula, $P$ and $Q, P$ and not- $P$ entails $Q$; and we will say a system is dialectical iff it is paraconsistent and yields (or "endorses") true contradictions, called "dialetheias". A paraconsistent system enables one to model theories, which, in spite of being (classically) inconsistent, are not trivial, while a dialectical system goes further, since it permits dialetheias, namely contradictions as true propositions. Still following Priest, the semantics of dialectical systems provides truth-value gluts (its worlds or set-ups are overdetermined); however, truth-value gaps (opened by worlds or set-ups which are underdetermined) are considered by Priest to be irrelevant or even improper for dialectical systems. Besides this, sometimes the distinction is drawn between weak and strong paraconsistency, the latter considered as equivalent with dialectics. A reader of recent literature in this field may have an impression that dialectics as strong paraconsistency is more a question of ontology than of logic itself, namely that it allows for the existence of "inconsistent facts" (in our actual world), which should verify dialetheias. One more introductory remark has to be made here: in recent literature on paraconsistent logic, there is some disagreement about the generally accepted distinction between paraconsistent and dialectical logical systems. However, it remains an open question whether semantic paradoxes express any "inconsistent facts".

A synthetic logic founded on rules 1, 2 and 3 must admit the truth of certain contradictions. If it is called dialectical, a logic that accepts that there are contradictions that may be true, then, in such a synthetic logic, there is a logical dialectic and meaning. The principle of totality, even at an elementary level, as here presented, necessarily leads to the principle of contradiction PNC. 


\section{The Opposition}

Some oppositions are indivisible data to thought, and their poles cannot be known in isolation but only with each other and in contrast, one to the other. These oppositions we will call logical dipoles. Thus, if we put a line and the displacement of a point along the line, the two possible directions of movement are identified immediately. Most logical categories are dipoles. Thus, it is impossible to define identity and difference separately, each being determined only by the negation of the other; true and false, necessary and contingent, etc. The logical importance of dipoles is that they limit analytical thinking, and its ability to divide and define in isolation. The same categories of logic are unnoticed in analytical logic, which applies them without being able to understand them because they are not analyzed. The dipole emerges in a synthetic logic or a dialectical logic.

Quality and quantity, logical categories, constitute a dipole. Quantitative differences are within a qualitative identity. Thus, the physical quantities (mass, temperature, electric charge, etc.) are qualities-quantities, that is, quantized qualities. Conversely, qualities are attributes that can present quantitative differences (bigger, smaller, equal), each being identical while having these differences. Positive, negative, rational and irrational are qualities of real numbers, for each of these attributes is indifferent to the magnitude of the number and encompass all quantitative comparisons between the numbers. Similarly, a function $f$ in $y=f(x)$ is the quality, and the difference between two functions $f$ and $g$ is qualitative because the function is identical to itself, though quantitative differences in the $x$ and $y$ variables show the differences.

Let $Q$ be a general quality.

Definition 1. When a general quality $Q$ subdivided is by means of elementary analysis into two particular subqualities $Q_{1}, Q_{2}$, these two subqualities are called opposite qualities, or poles of a qualitative opposition.

The identity or coincidence of the opposites $Q_{1}, Q_{2}$ is the general quality $Q$ that is common.

Definition 2. This general quality $Q$ will be called the unity of the opposition.

The idea of a coincidence of opposites is merely the expression of the neo-Platonic principle of the identity of the multiple. The Neoplatonists systems strove to show how from the most absolute unity, the One, the multiplicity of reality unfolds through a sliding scale. Each of the moments or steps of this scale represents a moment of greater dispersion relative to the upper rung, but it also implies a platform, where the realities of the bottom rung are unified and not yet dispersed. Looking at the scale from above, from the maximum unity of reality, each rung of the ladder successively shows a greater dispersion of the real; looking at the scale from below, from the maximum dispersion of sensible reality, each step up shows a greater degree of unity and concentration (coincidence) of reality. The maximum degree of unity corresponds to the first principle, the One. The coincidence of opposites signifies an understanding beyond the capacity of human reason. In response to this, Nicholas of Cusa distinguished two levels in knowledge or power, Reason and Understanding. Reason is governed by the principle of noncontradiction, which renders it incapable of understanding the coincidence of opposites: the PNC requires that something cannot be and not be at the same time, e.g., a line cannot be both curved and not curved, and, therefore, it is impossible to match the intended meeting of opposites. However, the supreme power does not depend on reason but on Understanding, and Understanding can gather the contraries or opposites that Reason declares incompatible (note that in general the opposites are not symmetrical). 
Example 1. Let 1 be the truth value indicating truth.

(1) The object is the set of microphysical corpuscles: as there are positive, negative, and positive and negative at the same time, and not electrified corpuscles, if one notes the proposition $P_{1}$ " $C$ is the electrified positive corpuscle" and the proposition $P_{2}$ "C is the electrified negative corpuscle", $P_{1}$ and $P_{2}$ are not logically coordinated.

(2) The object is the set of electrified corpuscles. Then, the true disjunction is $v\left(P_{1} \vee P_{2}\right)=1$. Opposites are disjunctive or sub-opposites.

(3) The object is the set of elementary microphysical corpuscles, i.e., none are positive or negative, that is, they are not electrified. Then, we will have true incompatibility $v\left(\neg P_{1} \vee \neg P_{2}\right)=1$. Opposites are incompatible or contrary.

(4) The object is the set of microphysical electrified elementary corpuscles. Then, we will have true mutual exclusion $v\left(P_{1} \Uparrow P_{2}\right)=1$. Opposites are exclusive or contradictory.

This example teaches us that as abstract analysis progresses, the opposite qualities, without logical coordination, are: sub-opposite (disjunctive), after opposite (incompatible) and ultimately contradictory.

Consider a whole with two opposite qualities $Q_{1}$ and $Q_{2}$ that are quantitatively uneven. The quantitative comparison is obviously founded on a common quality $Q$. Rule 3 concerning the resulting quality consists in attributing another quality to the whole, and by contributing the resulting quality, it is this quality which quantitatively dominates the other.

Definition 3. The whole is equipped with two opposing and quantitatively similar qualities. This equality defines the neuter of qualitative opposition.

Example 2. Suppose an elastic material link connecting two points $M$ and $N$. This link includes both an attraction and repulsion. When the distance $|M N|$ is greater than a certain value $r_{0}$, attraction dominates repulsion and the link is called attractive. It is called repulsive when $|M N|<r_{0}$ because then the repulsion is dominant. Therefore, in each case, the link does not stop being both attractive and repulsive, but rather has assigned to it the dominant quality.

The neuter is the case where $|M N|=r_{0}$, attraction and repulsion is then compensated exactly, and the link strength is zero. Under Rule 3, there is no attraction or repulsion, whereas under rule 2, attraction and repulsion continue working.

We see here that qualitative opposition, enriched by the neuter, leads to synthetic thought (dialectical), although here in this example, it is reduced to elementary forms.

\section{The True Contradictions}

We will present several cases of application of these rules to qualitative opposition.

\subsection{Application of Rules 1 and 2}

Take, for example, the Möbius surface. The analysis decomposes a strip into infinitesimal elements. All elements of non-zero curvature have a concave face and a convex face. Let $P_{01}$ and $P_{02}$ be the following propositions: $P_{01}=$ "the face $F$ of the element $E$ is concave", and $P_{02}=$ "the face $F$ of the element $E$ is convex". Considering the elements of zero curvature (neither concave nor convex) of Möbius strips, the incompatibility of $P_{01}$ and $P_{02}$ is true:

$$
v\left(\neg P_{01} \vee \neg P_{02}\right)=1
$$


According to Rule 1, we keep this analytic truth, but Rule 2 forces us to replace $P_{01}$ and $P_{02}$ " $E$ element" by "Möbius surface". $P_{01}$ and $P_{02}$, thus modified, are annotated $P_{1}$ and $P_{2}$, and we obtain, therefore, a first dialectical logic equation:

$$
v\left(\neg P_{1} \vee \neg P_{2}\right)=1
$$

Consider globally a Möbius surface that has only one face: $\mathrm{F}$ is the only face that is both concave and convex again, and here we always refer to rule 2 . We will have then:

$$
v\left(P_{1} \wedge P_{2}\right)=1
$$

It is this unique face, along a certain line of zero curvature, that is neither convex nor concave where

$$
v\left(\neg P_{1} \wedge \neg P_{2}\right)=1
$$

Finally, we get the system of logical equations:

$$
\left\{\begin{array}{l}
v\left(\neg P_{1} \vee \neg P_{2}\right)=1 \\
v\left(P_{1} \wedge P_{2}\right)=1 \\
v\left(\neg P_{1} \wedge \neg P_{2}\right)=1
\end{array}\right.
$$

and this system of equations is insoluble in classical logic as 4-1 and 4-2 are in themselves a contradiction, where the equivalence: $\neg P_{1} \vee \neg P_{2} \Leftrightarrow \neg\left(P_{1} \wedge P_{2}\right)$.

\subsection{Application of Rules 1, 2 and 3 to the Neuter of a Qualitative Opposition}

See Example 2: the analytical equation is $v\left(P_{01} \Im P_{02}\right)=1$ and not $v\left(\neg P_{01} \vee \neg P_{02}\right)=1$, $\Im$ being the complementarity between two propositions because there is a partial interaction of $\mathrm{M}$ with $\mathrm{N}$ that will be neither attractive nor repulsive. Rule 1 , corrected by Rule 2 , results in the formula $v\left(P_{1} \Uparrow P_{2}\right)=1$. Rule 2 always gives $v\left(P_{1} \wedge P_{2}\right)=1$. Rule 3 will be formulated as $v\left(P_{1}\right)=1$ if the quality is dominant and $v\left(\neg P_{1} \wedge \neg P_{2}\right)=1$ if it is a neutralized opposition. In the latter case, it is a system of logical equations such as:

$$
\left\{\begin{array}{l}
v\left(P_{1} \Im P\right)=1 \\
v\left(P_{1} \wedge P_{2}\right)=1 \\
v\left(\neg P_{1} \wedge \neg P_{2}\right)=1
\end{array}\right.
$$

\section{The Degree of Truth}

Let $p_{1}$ and $p_{2}$ be the truth values of $P_{1}$ and $P_{2}$, respectively. Consider system of Equations (5). It becomes the following system of algebraic equations [8]

$$
\left\{\begin{array} { l } 
{ p _ { 1 } + p _ { 2 } = 1 } \\
{ p _ { 1 } p _ { 2 } = 1 } \\
{ ( 1 - p _ { 1 } ) ( 1 - p _ { 2 } ) = 1 }
\end{array} \Rightarrow \left\{\begin{array}{l}
p_{1}+p_{2}=1 \\
p_{1} p_{2}=1 \\
p_{1}+p_{2}-p_{1} p_{2}=0
\end{array}\right.\right.
$$

This equation does not support any real solutions because $p_{1}$ and $p_{2}$ are roots of the equation $x^{2}-x+1=0$. The truth values in a multivalued logic are real numbers belonging to the interval [0,1] [9]. However, this multi-valued logic does not resolve the problem because it is essentially analytical. The equation $p^{2}-p+1=0$ is the characteristic equation of the true contradiction in a multi-valued logic. Indeed, if $P$ signifies any proposition whose truth value is $P$, its contradiction $\neg P$ has the truth-value $1-p$. The true contradiction is reflected in the logical equation $v(P \wedge \neg P)=1$ whose algebraic equation of truth values is $p(1-p)=1 \Rightarrow p^{2}-p+1=0$. System of Equations (5) expresses a contradiction in a dialectical logic based on the rules 1, 2 and 3 and has to be true. 
The equation $p^{2}-p+1=0$ has complex roots [8]:

$$
\left\{\begin{array}{l}
p^{1}=\frac{1}{2}+i \frac{\sqrt{3}}{2}=e^{i \frac{\pi}{3}} \\
p^{2}=\frac{1}{2}-i \frac{\sqrt{3}}{2}=e^{-i \frac{\pi}{3}}
\end{array}\right.
$$

This allows us to express the truth values using complex numbers.

Suppose the case where the quality contained in $P_{1}$ is dominant:

$$
\left\{\begin{array}{l}
v\left(P_{1} \Im P_{2}\right)=1 \\
v\left(P_{1} \wedge P_{2}\right)=1 \\
v\left(P_{1}\right)=1
\end{array}\right.
$$

It is possible to solve system of equations (8) taking truth values as modules of complex numbers (or the squares of these modules, in which nothing changes because it has truth values 0 and 1). Algebraically:

$$
\left\{\begin{array}{l}
\left|p_{1}+p_{2}\right|=1 \\
\left|p_{1} p_{2}\right|=1 \\
\left|p_{1}\right|=1
\end{array}\right.
$$

This system allows for the solution

$$
\left\{\begin{array}{l}
p_{1}=e^{i \frac{\pi}{3}} \\
p_{2}=e^{-i \frac{\pi}{3}}
\end{array}\right.
$$

Consider now the case where the analytical coordination of opposites is the disjunction. According to the rule that says when the analysis becomes increasingly less strict, and correspondingly the synthesis progresses, this coordination then passes from $v\left(P_{1} \Uparrow P_{2}\right)=1$ to $v\left(\neg P_{1} \vee \neg P_{2}\right)=1$ and then $v\left(P_{1} \vee P_{2}\right)=1$. It leads us to study the next case. This will result in the neuter of the opposition in the following system:

$$
\left\{\begin{array}{l}
v\left(P_{1} \vee P_{2}\right)=1 \\
v\left(P_{1} \wedge P_{2}\right)=1 \\
v\left(\neg P_{1} \wedge \neg P_{2}\right)=1
\end{array}\right.
$$

Both in multivalued logic and classical logic, incompatibility can be written as $v\left(\neg\left(P_{1} \wedge P_{2}\right)\right)=1$ or $v\left(\neg P_{1} \vee \neg P_{2}\right)=1$, these two compound propositions being equivalent. Therefore, Equation (4) can be written as:

$$
\left\{\begin{array}{l}
v\left(\neg P_{1} \vee \neg P_{2}\right)=1 \\
v\left(\neg P_{1} \wedge \neg P_{2}\right)=1 \\
v\left(P_{1} \wedge P_{2}\right)=1
\end{array}\right.
$$

The qualitative opposition and its neuter lead, in definition, to two basic types of systems of logic equations: systems of Equations (5) and (12); system of Equations (5) contains one contradiction and system of Equations (12) contains two.

However, in seeking solutions for this method, we reject multivalued logic because $p_{1}$ and $p_{2}$ are not the truth values of the propositions, but are complex numbers, determining by their modules these truth values. Therefore, we are entering a new logic.

\section{Dialectical Logic with Two Disjunctive Values and Complex Truth Values}

The logic presented here is multivalued: every proposition is equipped with a truth value in the interval [0,1] [9]. However, every truth value here is determined by a complex number that is the truth value of the proposition. 


\subsection{Truth Values}

To every proposition $P$ is assigned a truth value $p=|p| e^{i \alpha}$, with the condition $|p| \in[0,1]$. In the complex plane, there is a one-to-one correspondence between a proposition $P$ and a point $P$ of affix $\alpha$ located inside or over a circumference of the trigonometric circle. There is the same correspondence between a proposition $P$ and a free vector $\vec{p}$ of the plane, equipollent to $\overrightarrow{O P}, O$ being the center of the circle.

\subsection{Negation}

The truth value $\neg p$ of the negation $\neg P$ of the proposition $P$ is defined by the relationship

$$
|p+\neg p|=1
$$

Figure 1 shows that there is a set of negations $\{\neg P\}$ of the same proposition $P$, a set that has the power of the continuum.

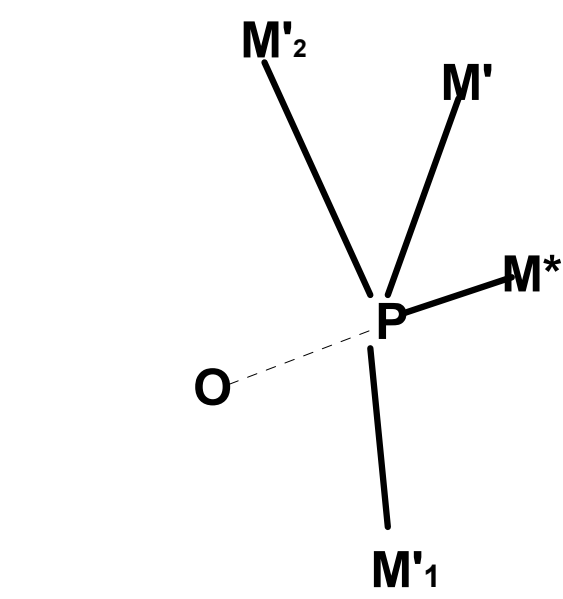

Figure 1. $\overrightarrow{P M^{\prime}}=\overrightarrow{p^{\prime}} ;\left|P M_{1}^{\prime}\right|=\left|P M_{2}^{\prime}\right|=1 ; P \vec{M}^{*}=\overrightarrow{\neg p}$.

The truth table for negation is shown in Table 1:

Table 1. Truth table for negation.

\begin{tabular}{cccc}
\hline $\boldsymbol{P}$ & T & TF & F \\
\hline$\neg P$ & F TF T & TF T & T \\
\hline
\end{tabular}

Consider, for example, the proposition $P=$ "it rains in the province of Alicante". If it is true, its negation $\neg P=$ "it does not rain in the province of Alicante" may be false if it has rained on the entire territory of the province, or be true if it has not rained in more than one part. The negation just defined makes sense in synthetic logic (Rules 1 and 2).

\subsection{Contradictory or Strong Negation}

The contradictory or strong negation of a proposition $P$, denoted $\neg P$, will for truth value, by definition, be $\neg p=(1-|p|) e^{i \alpha}$; in Figure 1 , it can be seen that $\neg P \in\{\neg P\}$.

The need to distinguish the contradictory of a proposition and its negation is already felt in Aristotelian logic - not when the content of the proposition belongs to a particular science but when it belongs to the same logic. Thus, $P_{1} \Leftrightarrow P_{2}$ is the negation of equivalence between $P_{1}$ and $P_{2}$, which can be either because there is no logical coordination or because they are linked by a coordination that is 
different from equivalence. Conversely, the contradiction of equivalence $\neg\left(P_{1} \Leftrightarrow P_{2}\right)$ is in Aristotelian logic a precise coordination: mutual exclusion $P_{1} \Uparrow P_{2}$.

The truth table for contradiction is shown in Table 2:

Table 2. Truth table for contradiction.

\begin{tabular}{|c|c|}
\hline$P$ & $10<|p|^{2}<10$ \\
\hline$\neg P$ & $0(1-|p|)^{2} 1$ \\
\hline
\end{tabular}

Returning to the previous example $P=$ "it rains in the province of Alicante", if this proposition is true, its contradiction can be spoken as "it does not rain in the province of Alicante" may not be false, since it can rain throughout the territory of the province or in a part only.

Coordination $P \Leftrightarrow \neg P$ is possible and occurs when $|p|=\frac{1}{2}$.

\subsection{Equivalence}

By definition, the equivalence of two propositions $\mathrm{P}_{1}$ and $\mathrm{P}_{2}$ is: $p_{2}=\left|p_{2}\right| e^{i \beta}$ and it is true when $\left|p_{1}\right|=\left|p_{2}\right|$

\subsection{Complementarity}

By definition, the compound proposition $P_{1} \Im P_{2}$ is called the complementarity of two propositions. $P_{1}$ and $P_{2}$ has the truth value $v\left(P_{1} \Im P_{2}\right)=\left|p_{1}+p_{2}\right|^{2}$ on the condition $\left|p_{1}+p_{2}\right| \leq 1$.

Complementarity is commutative. It may be false without either $P_{1}$ or $P_{2}$ being false. For this, it is necessary and sufficient for $|\alpha-\beta|=\pi$. $P_{1}$ and $P_{2}$ being true, it is true if $|\alpha-\beta|=\frac{2 \pi}{3}$.

The complementarity of $n$ propositions, with commutative and associative properties, has the truth value: $v\left(P_{1} \Im P_{2} \Im \ldots \Im P_{n}\right)=\left|p_{1}+p_{2}+\ldots+p_{n}\right|^{2}$ on the condition $\left|p_{1}+p_{2}+\ldots+p_{n}\right| \leq 1$.

From the definition of complementarity, it follows that $\forall P, v(P \Im \neg P)=1$.

\subsection{Conjunction}

By definition, the conjunction $P_{1} \wedge P_{2}$ has the truth value: $v\left(P_{1} \wedge P_{2}\right)=\left|p_{1} p_{2}\right|^{2}$. It has the commutative property. The combination of $n$ propositions has the truth value: $v\left(P_{1} \wedge P_{2} \wedge \ldots \wedge P_{n}\right)=$ $\left|p_{1} p_{2} \ldots p_{n}\right|^{2}$. It is commutative and associative.

\subsection{Weak Contradictions}

We will call weak contradictions the compound propositions $P \wedge \neg P$ with truth values $p *=|p||\neg p| e^{i\left(\alpha+\alpha^{\prime}\right)}$. Weak contradictions can be true. This rule is of great importance for synthetic or dialectic logic: $v(P \wedge \neg P)=1 \Rightarrow|p|=|\neg p|=1$. Figure 2 shows that the true contradiction has solutions:

$$
\begin{aligned}
& p=e^{i \alpha} \\
& \neg p=p e^{i\left(\alpha+\frac{2 \pi}{3}\right)} \\
& p+\neg p=e^{i \alpha\left(\frac{1}{2} \pm i \frac{\sqrt{3}}{2}\right)}
\end{aligned}
$$

We find the complex roots of -1 . 


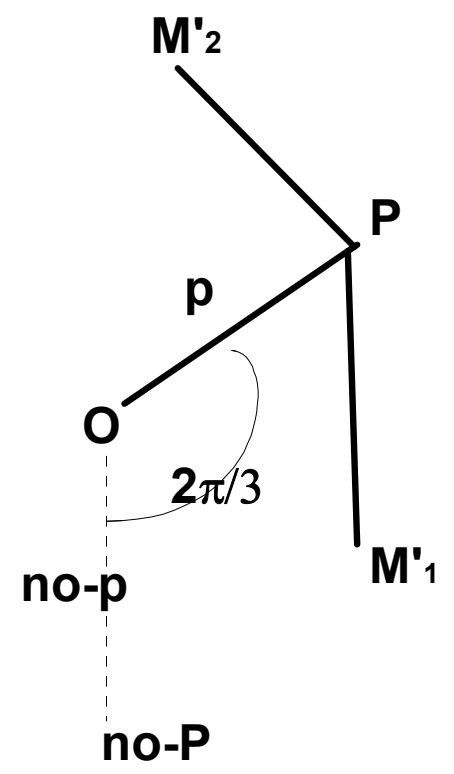

Figure 2. $\left|P M_{1}^{\prime}\right|=\left|P M_{2}^{\prime}\right|=1 ; p+\neg p=O M_{1}^{\prime} ; P \wedge \neg P=1$.

For example, the weak contradiction "it rains on the province of Alicante and it does not rain on the province of Alicante" is true if and only if it does not rain on more than a part of the province.

\subsection{Strong or Strict Contradiction}

We define as strong or strict contradiction the compound proposition $P \wedge \neg P$ with truth value $p *=|p|(1-|p|)$. As in Aristotelian logic, $|p|=0$ is false and $|p|=1$ is true. However, $|p|$ will never be true, and its maximum truth value is reached when $|p|=\frac{1}{2}$, that is, 0.0625 , i.e., is very weak.

Putting

$$
v(P \wedge \neg P)=0
$$

different truth values of 0 and 1 are excluded. Equation (13) is the equation of reduction of dialectical logic to Aristotelian logic, which is disjunctive, so $T$ and $F$ values become exclusive.

The strong contradiction "it rains on the province of Alicante and it does not rain all over the province of Alicante" is false in all cases.

\subsection{Incompatibility}

It is defined as a negation of the conjunction. If $\mu$ denotes a unitary complex number $\mu=e^{i \omega}, \omega$ having a value such that $\neg P_{1} \vee \neg P_{2}$ belongs to the set of negations of $P_{1} \wedge P_{2}$. Then,

$$
v\left(\neg P_{1} \vee \neg P_{2}\right)=\left|\mu-p_{1} p_{2}\right|^{2}
$$

\section{Resolution of Contradictions}

Let the system of Equations (5) be the system $\left\{\begin{array}{l}v\left(P_{1} \Im P\right)=1 \\ v\left(P_{1} \wedge P_{2}\right)=1 \\ v\left(\neg P_{1} \wedge \neg P_{2}\right)=1\end{array}\right.$. Only simple negations generate true contradictions, and we must eliminate the formulation of the system of Equations (5) strong negations. The algebraic version of the system of Equations (5) is then

$$
\left\{\begin{array}{l}
p_{1}+p_{2}=\mu_{1} \\
p_{1} p_{2}=\mu_{2} \\
\left(u_{1}-p_{1}\right)\left(u_{2}-p_{2}\right)=\mu_{3}
\end{array}\right.
$$


where $\mu_{1}, \mu_{2}$ and $\mu_{3}$ are the unit complex numbers of suitable arguments. $p_{1} p_{2}=\mu_{2}$ requires $\left|p_{1}\right|=\left|p_{2}\right|-1$ as true for $P_{1}$ and $P_{2}$. For the same reason, $\left(u_{1}-p_{1}\right)\left(u_{2}-p_{2}\right)=\mu_{3}$ requires that $\neg P_{1}$ is a true denial of $P_{1}$, and $\neg P_{2}$ is a true denial of $P_{2} \cdot p_{1}+p_{2}=\mu_{1}$ requires, as a consequence of $p_{1} p_{2}=\mu_{2}$, that $|\alpha-\beta|=\frac{2 \pi}{3}$. Graphically (see Figure 3):

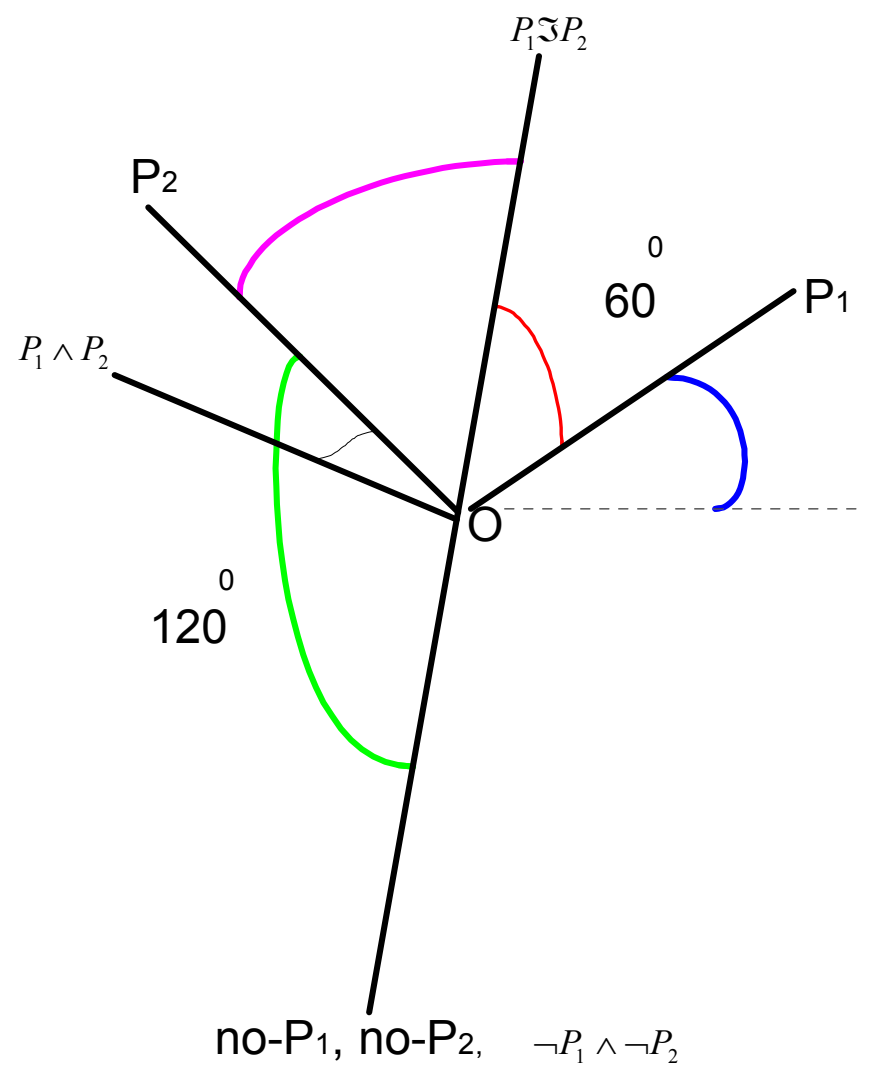

Figure 3. Resolution of contradictions.

Considering the system of Equations (11):

$$
\left\{\begin{array}{l}
v\left(P_{1} \vee P_{2}\right)=1 \\
v\left(P_{1} \wedge P_{2}\right)=1 \\
v\left(\neg P_{1} \wedge \neg P_{2}\right)=1
\end{array}\right.
$$

Its formulation in dialectical logic will be

$$
\left\{\begin{array}{l}
\mu_{0}-p_{1} p_{2}=\mu_{1} \\
p_{1} p_{2}=\mu_{2} \\
\left(u_{1}-p_{1}\right)\left(u_{2}-p_{2}\right)=\mu_{3}
\end{array}\right.
$$

$p_{1} p_{2}=\mu_{2}$ requires that $P_{1}$ and $P_{2}$ are true and $\left(u_{1}-p_{1}\right)\left(u_{2}-p_{2}\right)=\mu_{3}$ requires that $\neg P_{1}$ be a true negation of $P_{1}$ and that $\neg P_{2}$ be a true negation of $P_{2}$. $\mu_{0}-p_{1} p_{2}=\mu_{1}$ requires, as a consequence of $p_{1} p_{2}=\mu_{2}$, that arguments of $p_{1} p_{2}$ have a difference equal to $\pm \frac{2 \pi}{3}$. the system of Equations (11) is solved easily like the system of Equations (5).

\section{Conclusions}

Admitting the truth of a contradiction is extremely difficult for most of those who practice these parcels of knowledge that are called scientific. In spite of their understandable reluctance, we claim that 
classical logic never managed to oust contradiction from rational thought. Did not the Eleatics discover the famous antinomies that Aristotelian logic had failed to solve? Kant noted other contradictions hidden in the Newtonian concepts of space and time. In addition, modern mathematics, the theory of sets in particular, faces up to these contradictions, a problem which is catching the attention of logicians. While analytical logic has allowed and allows the development of particular scientific theories, its axioms insist that there be no contradiction. For this reason, it has been unable to resolve the logical and general problem of contradiction because, at some point in abstract analysis, analytical logic always gets caught in the antinomy trap.

The present authors are aware that the work is incomplete and plan to investigate further interesting perspectives. For example, opposition can be extended to opposition with neutrality. The degree of truth can be extended. The degree of falsehood may be studied as in intuitionistic fuzzy logic along with the degree of indeterminacy (or neutrality, i.e., neither true nor false) as in neutrosophic logic. Likewise, paradoxes such as the liar or Achilles and the tortoise may have different explanations using this logic. All of this can be developed in future work.

Acknowledgments: The authors like to thank Hugh Gash for his important collaboration in this work, especially English corrections and scientific recommendations.

Author Contributions: J.L.U.-D., J.A.N.-S. and L.S.-A. contributed to the theoretical development, and overall vision of the paper. J.L.U.-D. wrote the paper with J.A.N.-S.; J.A.N.-S. contributed writing, editing, and formatting of the paper.

Conflicts of Interest: The authors declare no conflict of interest.

\section{References}

1. Smarandache, F. Neutrosophy, A New Branch of Philosophy. Mult. Valued Logic 2002, 8, 297-384.

2. Smarandache, F. Law of Included Multiple-Middle E Principle of Dynamic Neutrosophic Opposition; Florentin Smarandache, Europa Nova \& Educational, Brussels-Columbus (Belgium-USA): Brussels, Belgium, 2014; p. 136.

3. Priest, G. In Contradiction. A Study of the Transconsistent; Martinus Nijhoff Publishers: Dordrecht, The Netherlands; Boston, MA, USA; Lancaster, UK, 1987.

4. Priest, G. Beyond the Limits of Thought; Cambridge University Press: Cambridge, UK, 1995.

5. Priest, G. Dialetheism; Stanford Encyclopedia of Philosophy: Stanford, CA, USA, 1998.

6. Priest, G.; Routley, R.; Norman, J. Paraconsistent Logic. Essays on the Inconsistent; Philosophia Verlag: Munchen, Germany; Hamden, CT, USA; Wien, Austria, 1989.

7. Priest, G.; Woods, J. Paraconsistency and Dialetheism. The Many Valued and Nonmonotonic Turn in Logic; Elsevier: Amsterdam, The Netherlands, 2007.

8. Nescolarde-Selva, J.; Usó-Doménech, J.L.; Alonso-Stenberg, K. An Approach to Paraconsistent Multivalued Logic: Evaluation by Complex Truth Values. In New Directions in Paraconsistent Logic; Springer: Kolkata, India, 2015; Chapter 6, pp. 147-163.

9. Usó-Doménech, J.L.; Nescolarde-Selva, J.; Pérez-Gonzaga, S. Truth values in T-norm based systems many-valued fuzzy logic. Am. J. Syst. Softw. 2014, 2, 139-145.

(c) 2016 by the authors; licensee MDPI, Basel, Switzerland. This article is an open access article distributed under the terms and conditions of the Creative Commons Attribution (CC-BY) license (http://creativecommons.org/licenses/by/4.0/). 